\title{
Variables sociofamiliares asociadas al abandono de los estudios universitarios
}

\section{Social and families variables associated with university drop-out}

\author{
Teresa GonzÁlez-Ramírez ${ }^{1}$ \\ tgonzale@us.es \\ Inmaculada Pedraza-Navarro \\ inmapednav@gmail.com \\ Universidad de Sevilla, España
}

\section{Resumen:}

Introducción. La finalidad fundamental de esta investigación es identificar las problemáticas socio-familiares que influyen en los estudiantes con riesgo de abandono en la universidad; analizando las relaciones existentes entre abandono universitario y variables como sexo, edad, nivel educativo y socioeconómico de las familias de origen. Método. Para ello hemos utilizado una metodología ex-post-facto, con un diseño descriptivo e inferencial. La muestra asciende a 86 estudiantes pertenecientes al Grado de Pedagogía y de Ciencias de la Actividad Física y del Deporte, de la Universidad de Sevilla que iniciaron sus estudios en el curso académico 2009/2010; no volviéndose a matricular, en los dos cursos siguientes. Para la recogida de datos se ha elaborado un cuestionario ad hoc con 51 ítems. En el análisis se han utilizado técnicas tanto descriptivas como de contraste. Resultados. Los resultados muestran cómo influye la presencia de problemáticas socio-familiares en los estudiantes provocando el abandono de-

\begin{abstract}
:
Introduction. The purpose of this research is to identify which social and family problems affect students at university dropout risk. In doing so, we will analyze the relationships between university drop-out and specific variables such as sex, age educational level and socio-economic status of parents. Method. We use an expost-facto methodology, with descriptive and inferential design. The sample is 86 students from the Degree of Pedagogy and Physical Activity and Sport at the University of Seville. These are students who began their studies in the academic year 2009/2010 and did not enrol again in this degree in the following two years. For data collection we developed an ad hoc questionnaire with 51 items. For the analysis we used descriptive and contrast techniques. Results. The results show how the existence of social and family problems affect students causing their drop out or temporary abandonment; at the same time, we detected which personal, family, institutional or social factors determine the
\end{abstract}

1 Dirección para correspondencia (correspondence address):

Teresa González-Ramírez. Departamento de Métodos de Investigación y Diagnóstico en Educación. Facultad de Educación. Universidad de Sevilla. C/ Pirotecnia, s/n. 41013 Sevilla (España). 
finitivo o transitorio; a la vez que hemos identificado qué factores personales, familiares, institucionales y sociales son determinantes en la decisión de abandonar una titulación. El $72 \%$ de la muestra presenta dificultades socio-familiares durante su transcurso académico, frente a un $26 \%$ que manifiesta desmotivación hacia los estudios que cursa y su preferencia por otra titulación o institución. Discusión. Estos resultados son concluyentes con investigaciones previas; en base a ello se realizan una serie de propuestas que puedan servir de reflexión a los responsables académicos para mejorar las tasas de éxito y rendimiento en las actuales titulaciones y propiciar una universidad más inclusiva.

\section{Palabras clave:}

Abandono; educación superior; problemáticas socio-familiares; cambio de titulación; calidad educativa. student's decision to drop out. $72 \%$ of the sample justified their drop-out in social and family variables while $26 \%$ alluded to demotivation and preference for another degree or institution. Discussion. These results coincide with previous research; in this way we make a series of proposals that can serve as a reflection for academic leaders to improve success rates and efficiency in current degrees and to promote an inclusive university.

\section{Key words:}

Drop-out; higher education; social and family problems; change of degree; educational quality.

\section{Résumé:}

Introduction. Le but de cette recherche est d'identifier quels problèmes sociaux et familiaux affectent les étudiants qui risquent d'abandonner leur université; analysant les relations entre le décrochage universitaire et les variables concrètes comme le sexe, l'âge, le niveau d'éducation et le statut socio-économique des parents. Méthode. Pour cela, nous utilisons une méthodologie ex-post-facto, avec une conception descriptive et inférentieIle. L'échantillon est composé de 86 étudiants de la Faculté de Pédagogie et D'activité Physique et Sportive de l'Université de Séville. Ce sont des étudiants qui ont commencé leurs études au cours de l'année universitaire 2009/2010, ne se tournant pas pour s'inscrire dans les deux années suivantes. Pour la collecte des données, nous avons élaboré un questionnaire ad hoc avec 51 items. Pour l'analyse, nous avons utilisé des techniques descriptives et de contraste. Résultats. Les résultats montrent comment l'existence de problèmes sociaux et familiaux affecte chez les élèves la désertion ou l'abandon temporaire; en même temps que nous avons détecté quels facteurs personnels, familiaux, institutionnels ou sociaux déterminent la décision de l'élève de décrocher un diplôme? $72 \%$ de l'échantillon montrent des variables sociales et familiales au cours de leur parcours scolaire, comparativement à $26 \%$ qui marquent leur démotivation et leur préférence pour un autre diplôme ou institution. Discussion. Ces résultats sont concluants avec des recherches antérieures; De cette façon nous faisons une série d'approche qui peut servir de réflexion pour les dirigeants universitaires afin d'améliorer les taux de réussite et l'efficacité dans les diplômes actuels. Encourager une université inclusive.

\section{Mots clés:}

Abandon; enseignement supérieur; problèmes sociaux et familiaux; changement de diplôme; qualité de l'éducation.

Fecha de recepción: 23-12-2016

Fecha de aceptación: 29-5-2017 


\section{Introducción}

El abandono de los estudios es actualmente un problema relevante y trascendente a nivel mundial por las múltiples consecuencias socioeconómicas que genera (Patrick, Shulenberg y O'Malley, 2016). La Organización para la Cooperación y Desarrollo Económico (OCDE, 2013) sitúa la tasa de abandono universitario alrededor del 30\%. Algunos países como EEUU o Italia llegan a medias del $55 \%$, y hasta de un $47 \%$ en América Latina (Moncada, 2014; Rojas Betancur, 2008). En España, el último informe del Ministerio de Educación, Cultura y Deporte (MECD, 2015) declara que el $19 \%$ de los estudiantes abandonan la universidad en su primer año ocasionando una pérdida anual de 1500 millones de euros para el gobierno español.

A nivel científico también es un tema que ha despertado un gran interés; existiendo actualmente un gran caudal de investigación que aborda esta problemática. Las líneas de investigación y avances se centran en cuantificar este fenómeno (Cabrera, Bethencourt, González y Álvarez, 2006a; García y Adrogué, 2015), construir y validar modelos explicativos del abandono (Cabrera, Bethencourt, Álvarez y González, 2006b; Schmitt y Santos, 2013), así como en identificar los factores asociados al mismo. Entre ellos, resulta necesario conocer las características personales y familiares de los estudiantes; e identificar las relaciones con su entorno socio-económico.

La identificación de las variables socio-familiares que pueden influir en el alumnado que decide abandonar es fundamental para comprender cómo "enganchar" al estudiante con sus estudios, prácticas y, por tanto, su desarrollo académico; con el objetivo de desarrollar propuestas educativas que mejoren la calidad del sistema universitario. Las universidades, acogidas a procesos de renovación de la acreditación deben velar por mejorar las tasas de rendimiento y éxito académico y reducir tanto el abandono transitorio como definitivo.

A continuación, presentamos el estado de la cuestión sobre la investigación realizada en torno a los factores explicativos del abandono universitario. 


\section{Marco teórico}

Una de las conclusiones fundamentales de la investigación actual es que el abandono es el resultado de una interacción de factores que desencadenan situaciones de riesgo, las cuales contribuyen a que el alumnado decida abandonar sus estudios (Elías, 2008). Distintas investigaciones (Cabrera y otros, 2006a; Lehmann, 2007; Bethencourt, Cabrera, Hernández, Álvarez y González, 2008; Castaño, Gallón, Gómez y Vásquez, 2008; Casquero y Navarro, 2010; Vries, León, Romero y Hernández, 2011; Aguilera y Jiménez, 2012; Severiens y ten Dam, 2012; García y Adrogué, 2015) analizan los factores personales, familiares, institucionales y sociales que actúan como variables intervinientes en el proceso educativo del estudiante; agrupándolas en variables personales del alumno y el contexto, vinculadas a las características del ambiente académico y social (Tejedor y García-Valcárcel, 2007; González, Álvarez, Cabrera y Bethencourt, 2007; Bethencourt y otros, 2008).

Para abordar esta multiplicidad, y acercarnos a una comprensión global y contextualizada de esta problemática se han sistematizado diferentes modelos y teorías explicativas que presentamos a continuación.

\section{Modelos teóricos explicativos del abandono en la universidad}

Las investigaciones realizadas sobre esta temática, se apoyan en diferentes modelos y teorías explicativas.

Cabrera y otros (2006b), los agrupan en cuatro grandes enfoques: Modelo de Adaptación, Modelo Estructural, Modelo Economicista y Modelo Psicopedagógico.

El modelo de adaptación considera que el abandono se produce debido a una desadaptación entre el sujeto y la cultura universitaria en la que éste se incorpora. Los trabajos de investigación realizados por Tinto (1975, 1987, 1989, 1993); Pascarella y Tenenzi (1991); Nora y Cabrera (1996); Koutsoubakis (1999); Hashway, Bahan, Hasway y Rogers (2000); Nora (2002); Metz (2002); Landry (2003) se encuadran en este modelo.

El modelo estructural concibe la deserción como el resultado de las contradicciones de los diferentes subsistemas (político, económico y social) que acaban influyendo en el individuo. Entiende que el abandono es un fenómeno inherente al sistema social en su conjunto, por lo que 
es difícil aislar los motivos específicos y reales que conducen a los estudiantes a la deserción. Entre las distintas investigaciones que se basan en este modelo destacan Bourdieu y Passeron (1977); Luján y Resendiz (1981); Thomas (2002).

El modelo economicista, basándose en la teoría del capital humano, postula que el abandono se debe a la elección por parte del estudiante de invertir su tiempo, energía y recursos en una forma alternativa que pueda producirle en el futuro mayores beneficios respecto a los costes de permanencia en la universidad (Schultz, 1961; Becker, 1962 y 1964; Thurow, 1973).

Finalmente, el modelo psicopedagógico, encuentra explicaciones al abandono en las características psicológicas y personales de los estudiantes; relacionándolo con variables de carácter psicopedagógico, tales como las estrategias de aprendizaje, la relación con el profesorado y compañeros, la habilidad para establecer y alcanzar metas o la capacidad para enfrentar los obstáculos y desempeñarse efectivamente en el ámbito académico. Las investigaciones realizadas por Cabrera y otros (2006b), a partir de los trabajos de Kirton (2000); Wasserman (2001) y Ryan y Glenn (2003) aportan que esas variables son determinantes en la decisión de abandonar o culminar con éxito los estudios.

Todos ellos, permiten ver el abandono desde una perspectiva institucional y a través de la trayectoria académica del estudiante.

Tendencias más actuales, abogan por la necesidad de aproximarnos a la problemática del abandono desde una perspectiva multidimensional. En este sentido, el modelo ecológico de Bronfenbrenner, es una alternativa integral para el estudio del abandono. Autores como Schmitt y Santos (2013) destacan la proyección que puede tener tanto a nivel teórico como empírico este modelo.

La teoría ecológica del desarrollo humano de Urie Bronfenbrenner, entiende que las personas se encuentran inmersas en un ambiente ecológico, entendido como las situaciones de contextos inmediatos o externos que influyen en el desarrollo del individuo; para Bronfenbrenner (1987), un medio ecológicamente compatible es aquel que se considera el más idóneo para el desarrollo del sujeto, entendiendo esta idoneidad como fruto del trabajo del sujeto, en construcción e interacción con ese entorno.

Para ello, establece cuatro sistemas: microsistema, mesosistema, exosistema y macrosistema. El microsistema comprende el nivel más cerca- 
no al individuo, abarca la familia, el aula, los compañeros de la escuela, los maestros y los vecinos. El mesosistema engloba las interrelaciones de dos o más ambientes en los que la persona se desenvuelve y realiza sus actividades tales como el hogar y los padres de familia que interaccionan para ayudar al individuo. El exosistema incluye ambientes más amplios en los cuales el individuo no está necesariamente activo. Por último, el macrosistema está formado por la cultura y subcultura en la que se desenvuelven todos los individuos de una misma sociedad. En su conjunto, representan una serie de estructuras contextuales concéntricas, que se relacionan entre sí y permiten comprender la incidencia de los contextos en el proceso formativo de los individuos.

Con el objetivo de sintetizar los modelos planteados, presentamos la figura 1.

\section{Modelo de adaptación}

-Desadaptación entre el sujeto y la cultura universitaria a la que se incorpora.

\section{Modelo estructural}

- Contradicciones de los diferentes subsistemas que influyen en el individuo.

\section{Modelo economicista}

- Alternativa de invertir tiempo, energía y recursos para producir futuros beneficios superiores respecto a costos en educación

\section{Modelo psicopedagógico}

- Explicaciones del abandono en las características psicológicas y personales del estudiante.

\section{Modelo ecológico}

- Sujetos se encuentran inmersos en un ambiente ecológico, entendido como situación de contextos inmediatos o externos que influyen en su desarrollo.

Figura 1. Síntesis sobre modelos explicativos del abandono universitario.

\section{Líneas de investigación sobre las variables que influyen en el abandono}

La revisión de la literatura sobre este tema nos ha permitido identificar tres líneas de investigación fundamentales: la primera de ellas hace referencia a la influencia que las variables personales tienen en el abandono universitario (Cabrera y otros, 2006a; Bethencourt y otros, 2008; Elías, 2008; Lassibille y Navarro Gómez, 2008; Gury, 2011; Bernardo, 
Cerezo, Rodríguez-Muñíz, Núñez, Tuero y Esteban, 2015; Clerici, Giraldo y Meggiolario, 2015; García y Adrogué, 2015; Roso-Bas, Pades Jiménez y García-Buades, 2016). La segunda se centra en la relación entre variables personales e institucionales (Tejedor y García-Varcalcel, 2007; Álvarez y López, 2011; Belloc, Maruotti y Petrella, 2011; Vries y otros, 2011; Aguilera y Jiménez, 2012; Whannel, 2013; Bernardo y otros, 2015; García y Adrogué, 2015). La tercera en la relación entre variables psico-educativas y variables socio-políticas (Cabrera y otros, 2006a; Lehmann, 2007; Castaño y otros, 2008; Casquero y Navarro, 2010; Gury, 2011; Severiens y ten Dam, 2012; Whannel, 2013; Heublein, 2014; García y Adrogué, 2015).

\section{Línea de investigación sobre las variables personales que influyen en el abandono}

Los trabajos que se han realizado dentro de esta línea de investigación, vinculan el abandono con la edad temprana en la que se realiza esa elección y la falta de información para tomar la decisión adecuada (García y Adrogué, 2015).

A su vez, la elección de una titulación se ve influenciada por la nota de acceso que demanda cada institución. Cuando el estudiante no posee la nota de corte requerida se ve obligado a matricularse en una titulación o institución que no es de su preferencia. Elías (2008) en su estudio concluye que los estudiantes dejan la carrera al no ser ésta su primera opción de pre-inscripción. En este sentido, poseer mejores calificaciones en bachillerato reduce significativamente los riesgos de abandono en la universidad (Gury, 2011; Bernardo y otros, 2015). Por tanto, el rendimiento académico previo se presenta como una variable que influye en el abandono.

Otra variable que también influye en el abandono es el modo de acceso; el trabajo realizado por Clerici y otros, (2015) muestra que los estudiantes que entran en la universidad mediante grados de formación profesional son más propensos a abandonar que los que provienen de Educación Secundaria al no estar suficientemente formados en las competencias básicas para tener éxito en la educación superior (Lassibille y Navarro Gómez, 2008).

A estos conocimientos previos, se unen la motivación hacia la titulación o institución elegida y las características psicológicas de los estu- 
diantes (Cabrera y otros, 2006a; Bethencourt y otros, 2008). Tener una alta autoestima, ayuda a poner énfasis en las fortalezas, a eliminar pensamientos negativos al enfrentarse a nuevos desafíos. Otra conclusión de la investigación, dentro de esta línea es que aquellos individuos con una alta autoestima son más propensos a continuar sus estudios (Roso-Bas y otros, 2016).

\section{Línea de investigación sobre la relación entre variables personales e institucionales que influyen en el abandono}

Los estudiantes que asisten con frecuencia a clase tienen mayor probabilidad de permanecer en la universidad (Bernardo y otros, 2015; Whannel, 2013). La asistencia a clase favorece el establecimiento de relaciones entre los distintos agentes, el compromiso emocional y la identidad, las horas de estudio y la autoeficacia académica (Nora, 2001; Álvarez y López, 2011) mejorando el rendimiento académico de los estudiantes.

Según Tejedor y García-Varcarcel (2007) existen una serie de causas: a) relacionadas con los aspectos organizativos de la institución de acogida (número de asignaturas, extensión de los programas, dificultad de las materias, recursos para la docencia, ratio profesor/alumno, horario de las clases, número de clases prácticas, número de exámenes y trabajos, clima institucional), b) relacionadas con el profesor (excesiva exigencia, tipo de exámenes, adecuación de las pruebas de evaluación, subjetividad en la corrección de los exámenes, escasa información sobre los criterios de evaluación, comunicación profesor-estudiante) y c) relacionadas con el estudiante (dominio de técnicas de estudio, responsabilidad y nivel de exigencia, absentismo-participación-asistencia, clima de clase) que influyen en el rendimiento de los estudiantes. Estos problemas de rendimiento se ven acompañados de inadecuadas condiciones de estudio (Belloc y otros, 2011) y problemas en la gestión del tiempo.

Algunos estudiantes, deciden dedicar su tiempo y esfuerzo en alternativas que le proporcionen mayor beneficio, como incorporarse al mundo laboral. En este sentido, Vries y otros (2011); Aguilera y Jiménez (2012) destacan la incompatibilidad de horas de estudio con horas de trabajo. Según García y Adrogué (2015) es mayor el riesgo de abandono en los estudiantes que trabajan. 


\section{Línea de investigación sobre la relación entre variables psico-educativas y variables socio-políticas}

En palabras de Whannell (2013) la deserción involucra esencialmente a los estudiantes más jóvenes. El aumento de edad decrece el riesgo de desertar; siendo mayor el riesgo de abandono en los hombres, así como, entre casados (Castaño y otros, 2008; García y Adrogué, 2015).

El tamaño de la familia también se presenta como variable relacionada con el abandono. Según Casquero y Navarro (2010) cuanto mayor es el número de hermanos de 16 años que estudian, menor es el riesgo de desertar. Este riesgo aumenta al vivir fuera del entorno familiar, y ante la presencia de dificultades familiares (Cabrera y otros, 2006a). Algunos estudiantes presentan dificultades para combinar cuidado de los niños o cuidado de otros miembros de la familia con sus estudios (Heublein, 2014; Alonso-Sanz, Iglesias Martínez y Lozano Cabezas, 2015). Especialmente se produce entre las mujeres. Ante la presencia de dificultades se observa distinción entre sexos. Severiens y ten Dam (2012) asiente que el abandono por parte de las mujeres se debe principalmente a factores familiares tales como el nacimiento de hijos, matrimonio o presencia de enfermedades.

Pertenecer a un estrato social inferior también influye negativamente en el desempeño académico (Lehmann, 2007). A menor nivel socio-económico y socio-educativo mayor probabilidad de abandono (Castaño y otros, 2008; García y Adrogué, 2015). Gury (2011) muestra que aquellos estudiantes cuyos padres tienen trabajos manuales son más propensos a abandonar. La situación de la madre, en este sentido, no ejerce ningún impacto. Y señala que los padres con una buena educación incitan a los estudiantes a continuar sus estudios con el fin de que consigan graduarse. Cuanto mejor es el clima educativo del hogar menor es la probabilidad de abandonar (García y Adrogué, 2015).

En síntesis, estas investigaciones ofrecen un amplio panorama sobre la complejidad del abandono. La multiplicidad de variables personales, familiares, institucionales y sociales interrelacionadas entre sí, desencadenan situaciones de riesgo que contribuyen a la decisión del alumnado de abandonar sus estudios.

Conocer qué tipo de variables influye en los estudiantes durante su proceso educativo permite determinar la naturaleza del abandono; por lo que es necesario continuar el análisis de forma científica y empírica. 


\section{Objetivos e Hipótesis}

El objetivo fundamental de esta investigación es identificar las problemáticas socio-familiares que influyen en los estudiantes con riesgo de abandono universitario; para analizar la relación existente entre:

- Riesgo de abandono universitario y edad de los estudiantes.

- Riesgo de abandono universitario y sexo de los estudiantes.

- Riesgo de abandono universitario y nivel educativo de los padres.

- Así como, riesgo de abandono universitario y nivel socio-económico de los mismos.

Para ello, formulamos distintas hipótesis nulas y alternativas, que declaramos en los siguientes términos:

- $\mathrm{H}^{0}$ (Hipótesis nula): No existen diferencias significativas entre el riesgo de abandono universitario y las variables concretas analizadas, con un riesgo alfa de equivocarnos del .05.

- $\quad H^{1}$ (Hipótesis alternativa): Sí existen diferencias significativas entre el riesgo de abandono universitario y las variables concretas analizadas, con un riesgo alfa de equivocarnos del .05.

\section{Metodología de la investigación}

Este estudio se encuadra en una metodología ex-post-facto, con un diseño descriptivo e inferencial.

\section{Población y muestra}

La población objeto de estudio, la conforman dos grupos de estudiantes que han estado matriculados en la Facultad de Ciencias de la Educación, de la Universidad de Sevilla. El primer grupo corresponde a estudiantes del Grado de Ciencias de la Actividad Física y el Deporte y el segundo al Grado de Pedagogía. En total constituyen una población de 110 estudiantes; de los cuales 14 pertenecen al Grado de Ciencias de Actividad Física y Deporte, y 96 al Grado de Pedagogía. Ambos grupos iniciaron sus estudios en el curso académico 2009/2010 abandonándolos; no volviéndose a matricular en los dos cursos siguientes. 
A partir de cómo queda definido el indicador abandono inicial en el Sistema de Garantía de la Calidad de la Universidad de Sevilla ${ }^{1}$, en el grado de Pedagogía la población total de alumnado, que según el criterio de abandono resulta, es de 96 estudiantes de una cohorte de entrada de 240; de la cual se consigue contactar con 75. En el grado de Ciencias de la Actividad Física y del Deporte de una cohorte de entrada de 60, se contacta con 11 estudiantes; quedando en este caso la muestra objeto de estudio, configurada por 86 estudiantes pertenecientes a ambas titulaciones.

La Tabla 1 aporta esta información:

Tabla 1. Número de estudiantes que conforman la tasa de abandono de los Grados de Ciencias de la Actividad Física y del Deporte y Pedagogía.

\begin{tabular}{|c|c|c|c|}
\hline \multirow{2}{*}{\multicolumn{2}{|c|}{$\begin{array}{l}\text { Grado de CAFD } \\
\text { De una cohorte de nuevo ingreso de } \\
60 \text { estudiantes en el año 2009/2010, } \\
14 \text { son los estudiantes que no se ma- } \\
\text { tricularon en los cursos académicos } \\
2010 / 2011 \text { y } 2011 / 2012 .\end{array}$}} & \multicolumn{2}{|l|}{ Grado de Pedagogía } \\
\hline & & $\begin{array}{l}\text { De una cohorte de } \\
240 \text { estudiantes en } \\
96 \text { son los estudiant } \\
\text { tricularon en los cur } \\
2010 / 2011 \text { y } 2011 / 2\end{array}$ & $\begin{array}{l}\text { luevo ingreso de } \\
\text { l año 2009/2010, } \\
\text { es que no se ma- } \\
\text { sos académicos } \\
012 .\end{array}$ \\
\hline $\begin{array}{c}\text { Cohorte de } \\
\text { nuevo ingreso } \\
(2009 / 2010)\end{array}$ & 60 estudiantes & $\begin{array}{c}\text { Cohorte de } \\
\text { nuevo ingreso } \\
(2009 / 2010)\end{array}$ & 240 estudiantes \\
\hline $\begin{array}{c}\text { No matriculación } \\
\text { curso } 2010 / 2011 \\
\text { y } 2011 / 2012 \\
\end{array}$ & 14 estudiantes & $\begin{array}{c}\text { No matriculación } \\
\text { curso } 2010 / 2011 \\
\text { y } 2011 / 2012 \\
\end{array}$ & 96 estudiantes \\
\hline
\end{tabular}

En términos de porcentaje, la tasa de abandono del Grado de Ciencias de la Actividad Física y del Deporte es del 23.3 \% y la del Grado de Pedagogía es del 40\%. La figura 2 permite visualizar estos datos:

\begin{tabular}{llll}
\hline \multicolumn{2}{c}{ Grado de CAFD } & Grado de Pedagogía \\
\hline & $23.3 \%$ & & \\
\hline & & & \\
\hline $76.7 \%$ & $60 \%$ & $40 \%$ \\
\hline
\end{tabular}

$\%$ alumnado que continúa estudios $\square \%$ alumnado que abandona estudios

Figura 2. Porcentaje de abandono en el Grado de Ciencias de la Actividad Física y del Deporte y el Grado de Pedagogía (cohorte 2009-2010). 


\section{Datos sociológicos de la muestra}

Con el fin de sintetizar el perfil sociológico de los estudiantes que constituyen la muestra presentamos la tabla 2.

Tabla 2. Síntesis del perfil sociológico de los estudiantes con riesgo de abandono universitario que constituyen la muestra.

\begin{tabular}{|c|c|c|}
\hline \multicolumn{2}{|l|}{ Grado de CAFD } & Grado de Pedagogía \\
\hline Hombre & Sexo & Mujer \\
\hline 22 & Edad Media & 24 \\
\hline Solteros & Estado civil & Solteros \\
\hline Sevilla & Residencia actual & Sevilla \\
\hline $\begin{array}{l}\text { Alquiler } \\
\text { Familiar }\end{array}$ & Tipo de vivienda & Familiar \\
\hline Inactivo & Situación laboral & Inactivo \\
\hline $\begin{array}{l}\text { Ayuda familiar } \\
\text { Ayuda MECD }\end{array}$ & Financiación de estudios & $\begin{array}{l}\text { Ayuda familiar } \\
\text { Ayuda MECD }\end{array}$ \\
\hline Estudios universitarios & Formación de padres & $\begin{array}{l}\text { Primarios } \\
\text { Secundarios }\end{array}$ \\
\hline Grupo 2 & Profesión de padres (CIOU) & Grupo 2, 3 y 4 \\
\hline Padre + Madre & Sustentador familiar & Padre \\
\hline 1 & $\begin{array}{c}N^{\circ} \text { de hermanos que residen } \\
\text { en la unidad familiar }\end{array}$ & 1 \\
\hline $\begin{array}{l}\text { En formación } \\
\text { Estudios universitarios }\end{array}$ & Formación de los hermanos & $\begin{array}{c}\text { En formación } \\
\text { Estudios universitarios- } \\
\text { técnicos }\end{array}$ \\
\hline
\end{tabular}

\section{Recogida de datos}

Para la recogida de datos se ha elaborado un cuestionario, que responde de forma precisa al objetivo científico e hipótesis planteadas. La revisión de la bibliografía efectuada sirve de referencia para su elaboración.

Este cuestionario está compuesto por 51 ítems estructurados en cuatro dimensiones:

- Datos de identificación, compuesto por 6 ítems.

- Contexto socio-cultural a partir de 9 ítems.

- Trayectoria universitaria del estudiante, formado por 20 ítems. Para medir cada uno de los ítems se ha utilizado una escala tipo Lickert de 0 a 5 puntos. 
- Problemáticas socio-familiares asociadas al alumnado compuesta por 16 ítems.

Para analizar los datos se ha utilizado el software estadístico SPSS v.22.

\section{Resultados y discusión}

\section{Incidencia de problemáticas socio-familiares en los estudiantes con riesgo de abandono universitario}

Dando respuesta a nuestro objetivo científico presentamos a continuación, las problemáticas que influyeron en los estudiantes durante su transcurso universitario. Ante la no presencia de estas, mostraremos el motivo que les impulsó a abandonar la titulación.

De los 86 estudiantes que conforman la muestra, el $72 \%$ de los encuestados presentan algún tipo de problemática (62 estudiantes). Entre las problemáticas socio-familiares que señalan observamos:

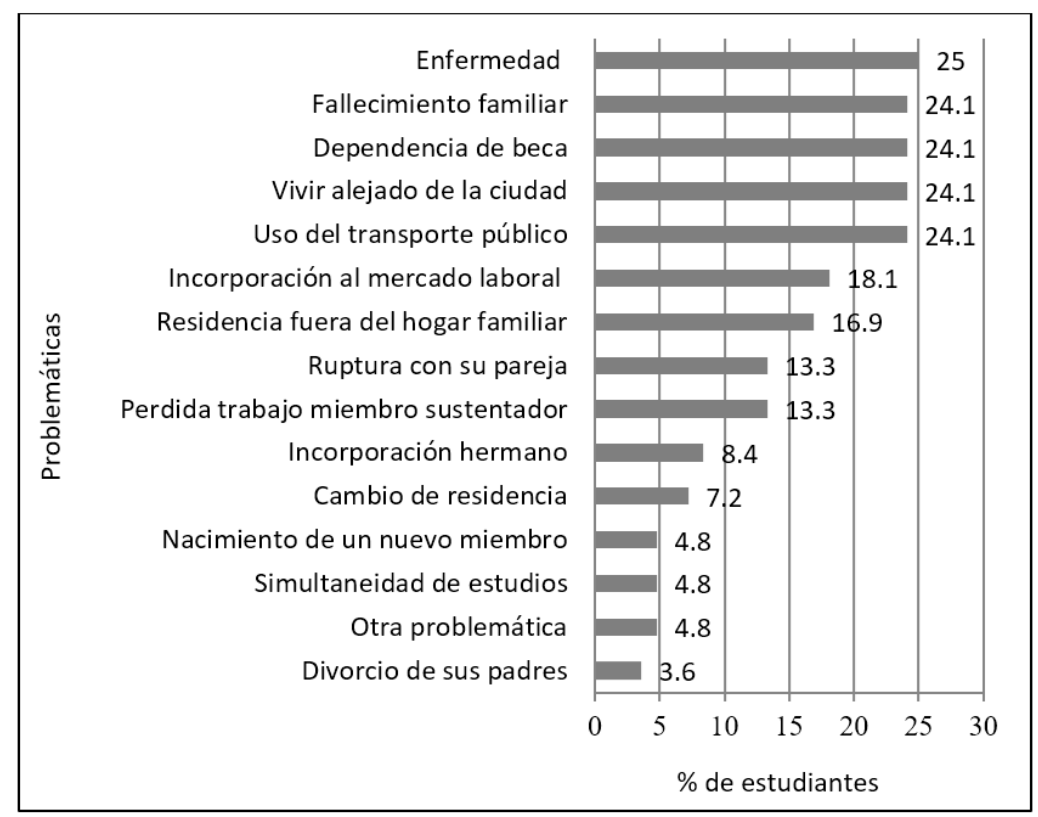

Figura 3. Incidencia de problemáticas socio-familiares en los estudiantes. 
Analizando la figura 3, vemos cómo "enfermedad propia o de algún familiar" (25\%) presenta un porcentaje mayor, seguido de "fallecimiento de un familiar o persona cercana", "vivir alejado de la ciudad", "uso del transporte público" y "dependencia de beca" (24.1\%). En menor medida se encuentra "incorporación al mercado laboral" (18.1\%), "residencia fuera del hogar familiar" (16.9\%), "perdida de trabajo del miembro sustentador de la familia" y "ruptura con su pareja" (13.3\%). En un tercer nivel se muestran problemáticas vinculadas a "incorporación de un hermano a la universidad" (8.4\%), "cambio de residencia durante el transcurso del año" (7.2\%), "simultaneidad de estudios" y "nacimiento de un nuevo miembro en la familia" $(4.8 \%)$. Con un porcentaje menor "Divorcio de sus padres u algún familiar cercano" (3.6\%).

Entre el $26 \%$ de la muestra que no presenta ningún tipo de problemáticas (22 estudiantes), los motivos que propiciaron su abandono fueron:

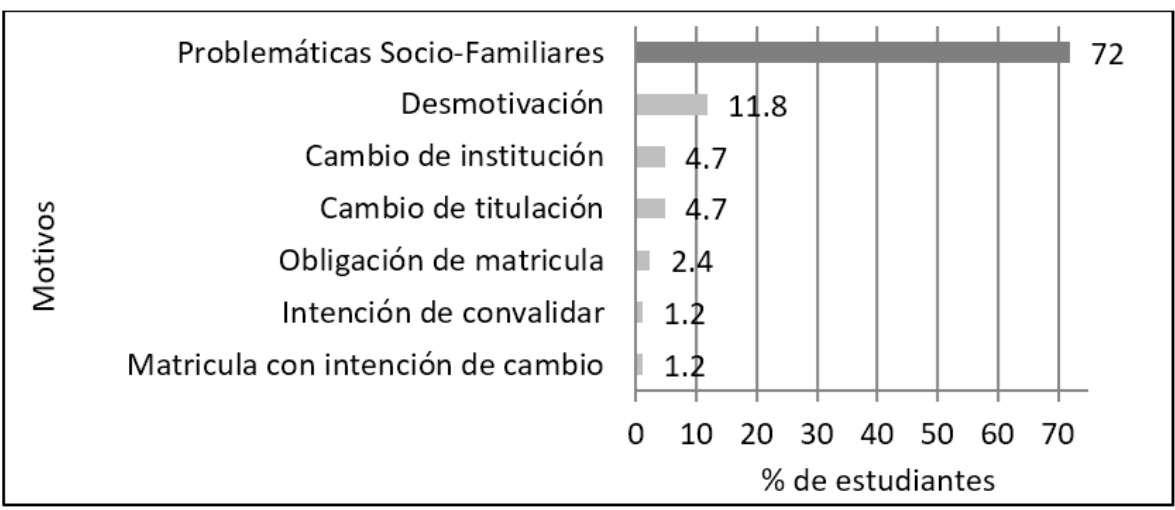

Figura 4. Motivos de abandono de los estudiantes ante la no presencia de problemáticas socio-familiares.

En la figura 4, observamos cómo en aquellos estudiantes que no presentan problemáticas familiares, la decisión de abandonar fue originada por desmotivación con los estudios (11.8\%). De igual manera estos resultados muestran el porcentaje de estudiantes que realizaron un cambio de titulación durante el mismo curso académico o incluso antes de comenzar $(4.7 \%)$. En menor rango se encuentran los estudiantes que realizan el primer año de titulación, debido a la pérdida del plazo de matrícula $(2.4 \%)$, formalizar una matrícula de primer año con la intención de convalidar asignaturas para poder realizar en el curso siguiente 
la titulación deseaba (1.2\%); y finalmente otro $1.2 \%$ cuyo objetivo es matricularse para cambiarse el curso siguiente.

\section{Contraste de hipótesis}

Con el fin de analizar las relaciones existentes entre el riesgo de abandono universitario y las variables concretas analizadas (edad, sexo del estudiante, nivel educativo y socio-económico de los padres) a continuación presentamos los resultados obtenidos en términos de contraste de hipótesis.

Para ello hemos utilizado el test estadístico de $U$ de Mann-Whitney y la Chi-cuadrado de Pearson según las características específicas de los datos obtenidos.

1. Relación entre riesgo de abandono universitario y edad de los estudiantes:

Este primer contraste lo realizaremos respecto a la edad de los estudiantes.

Tras efectuar el test estadístico de $U$ de Mann-Whitney, observamos un nivel de significatividad de .829. Nos lleva a afirmar la hipótesis nula y rechazar la hipótesis alternativa. A nivel poblacional, no existen diferencias significativas entre el riesgo de abandono universitario y la edad de los estudiantes, es decir, ambas variables son independientes entre sí.

2. Relación entre riesgo de abandono universitario y sexo de los estudiantes:

A continuación, pasamos a analizar si existen diferencias significativas entre riesgo de abandono universitario y sexo de los estudiantes. Para ello, efectuamos la prueba de Chi-cuadrado de Pearson y se observa un nivel de significatividad de .002. Ésta nos lleva a rechazar la hipótesis nula y afirmar la hipótesis alternativa. Existe relación entre las dos variables.

Tal y como muestra la figura 5, de un total de 86 estudiantes encuestados que abandonan, 62 son de sexo "mujer" (72.1\%) frente a 24 de sexo "hombre" (27.9\%). Se hace visible qué es más frecuente el abandono por parte de las mujeres. 


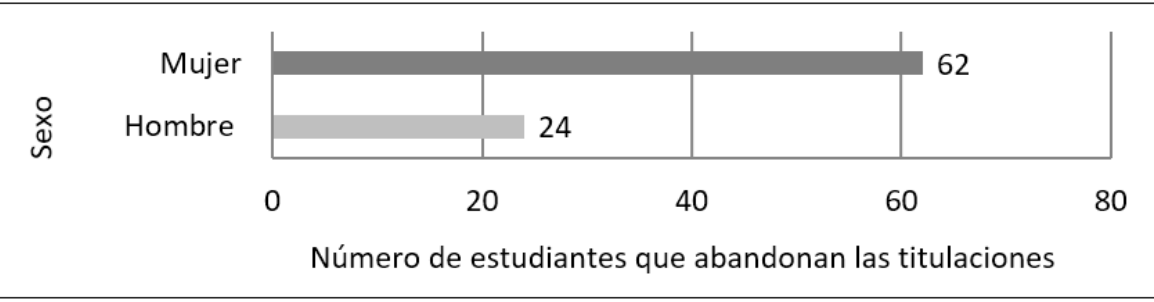

Figura 5. Sexo de los estudiantes.

A su vez, es el sexo mujer quien señala mayor presencia de problemáticas socio-familiares a lo largo de su transcurso académico. Si analizamos la figura 6 que presentamos a continuación, podemos ver que el sexo mujer presenta mayor frecuencia en todas las problemáticas.

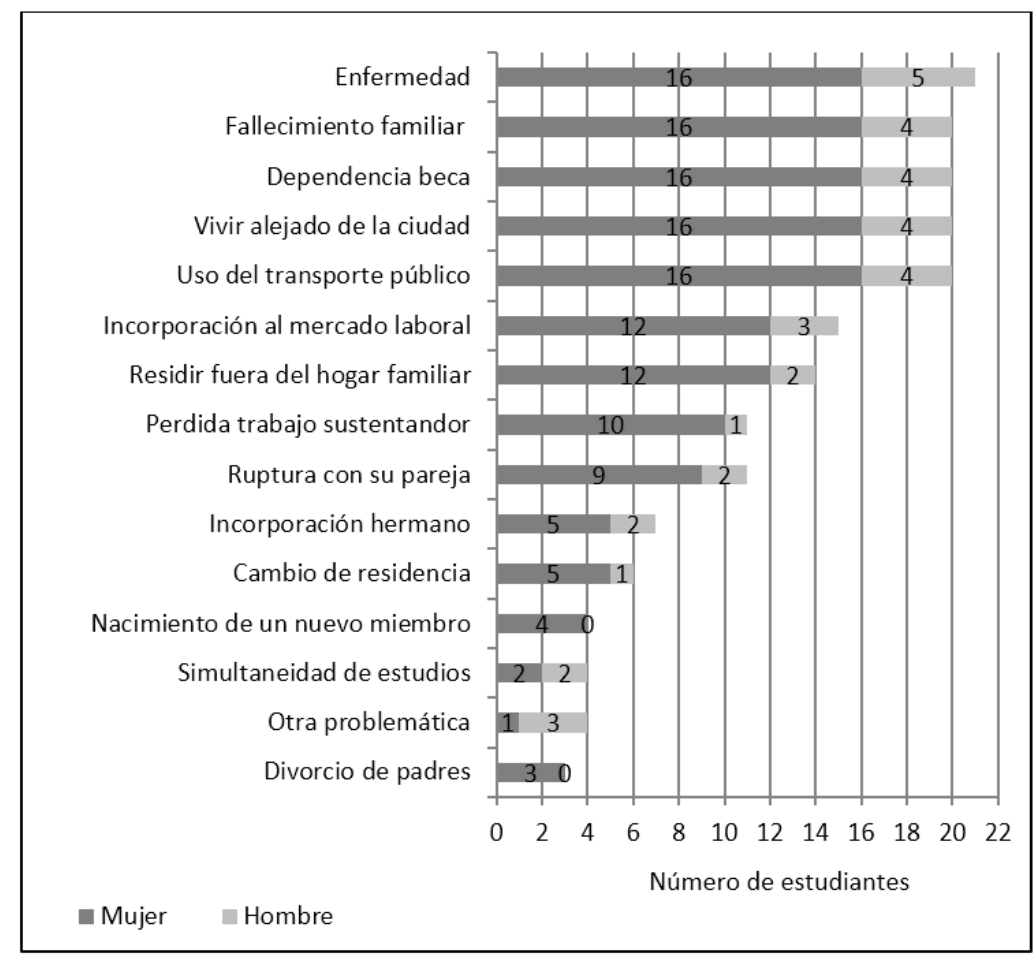

Figura 6. Presencia de problemáticas socio-familiares en los estudiantes en función de su sexo.

Existen dos problemáticas propias del sexo mujer: "nacimiento de un nuevo miembro en la familia" y "divorcio de los padres". 
Entre "otras problemáticas", los estudiantes destacan: simultanear trabajo con estudios y cuidar de un familiar.

Existe relación significativa entre sexo y otras problemáticas. Tras realizar una tabla de contingencia con ambos ítems y efectuar la prueba de Chi-cuadrado de Pearson, se observa un nivel de significatividad de .030. Se rechaza la hipótesis nula y se afirma la hipótesis alternativa. Existen diferencias significativas entre el sexo y otras problemáticas, es decir, existe relación entre ambas variables.

3. Relación entre riesgo de abandono universitario y nivel educativo de los padres:

El nivel educativo de los padres se mide mediante dos ítems: "formación académica del padre" y "formación académica de la madre". Así, para analizar la relación existente entre el riesgo de abandono universitario y el nivel educativo de los padres analizamos cada uno de estos ítems por separado.

Centrándonos en la figura del padre, tras realizar una tabla de contingencia y efectuar la prueba de Chi-cuadrado de Pearson, observamos un nivel de significatividad de .119. Afirmamos la hipótesis nula; no existen diferencias significativas entre el riesgo de abandono universitario y la formación académica del padre.

En relación a la formación académica de la madre, tras efectuar nuevamente una tabla de contingencia y la prueba de Chi-cuadrado de Pearson, observamos un nivel de significatividad de .184. Afirmamos la hipótesis nula; no existen diferencias significativas entre el riesgo de abandono universitario y la formación académica de la madre. Ambas variables son independientes entre sí.

4. Relación entre riesgo de abandono universitario y nivel socio-económico de los padres:

El nivel socio-económico de los padres atiende a dos ítems: "profesión actual de los padres" y "profesión actual de las madres". Para medir las diferencias significativas entre riesgo de abandono universitario y nivel socio-económico de los padres debemos atender a cada uno de estos ítems por separado.

Centrándonos en la figura del padre, tras realizar una tabla de contingencia y efectuada la prueba de Chi-cuadrado de Pearson, observamos un nivel de significatividad de .430. Afirmamos la hipótesis nula; no 
existen diferencias significativas entre el riesgo de abandono universitario y la profesión actual de los padres.

En relación a la profesión actual de madre, tras realizar la tabla de contingencia y la prueba de Chi-cuadrado de Pearson, observamos un nivel de significatividad de .034. Rechazamos la hipótesis nula y afirmamos la hipótesis alternativa. Existen diferencias significativas entre ambas variables.

Tras analizar el perfil profesional de las madres, podemos decir que la elevada cifra de madres amas de casa (33.7\%) y en desempleo (10.8\%), son características que influyen negativamente en el abandono universitario de los estudiantes.

Este nivel socio-económico de los padres está íntimamente relacionado con su nivel educativo. Realizando un análisis de contraste entre ambas variables observamos un nivel de significatividad de .023 mediante la prueba de Chi-cuadrado de Pearson.

A modo síntesis, la tabla 3 resume brevemente los resultados alcanzados y las hipótesis aceptadas en nuestro estudio.

Tabla 3. $\mathbf{H}^{0}$ y $\mathbf{H}^{1}$ aceptadas en los diferentes contrastes realizados.

\begin{tabular}{lccc}
\hline Relación de variables & Valor & $\begin{array}{c}\text { Nivel de } \\
\text { Significatividad }\end{array}$ & $\begin{array}{c}\text { Hipótesis } \\
\text { aceptada }\end{array}$ \\
\hline $\begin{array}{l}\text { Entre riesgo de abandono universitario } \\
\text { y edad de los estudiantes. }\end{array}$ & .829 & $\mathrm{H}^{0}$ \\
\hline $\begin{array}{l}\text { Entre riesgo de abandono universitario } \\
\text { y sexo de los estudiantes. }\end{array}$ & 19.122 & .002 & $\mathrm{H}^{1}$ \\
$\begin{array}{l}\text { - Ante la presencia de otras problemá- } \\
\text { ticas. }\end{array}$ & 4.691 & .030 & $\mathrm{H}^{1}$ \\
\hline $\begin{array}{l}\text { Entre riesgo de abandono universitario } \\
\text { y nivel educativo de los padres. }\end{array}$ & & & \\
$\begin{array}{l}\text { - Atendiendo a la formación académi- } \\
\text { ca del padre. }\end{array}$ & 21.584 & .119 & $\mathrm{H}^{0}$ \\
$\begin{array}{l}\text { - Atendiendo a la formación acadé- } \\
\text { mica de la madre. }\end{array}$ & 19.705 & .184 & $\mathrm{H}^{0}$ \\
$\begin{array}{l}\text { Entre riesgo de abandono universitario } \\
\text { y nivel socio-económico de los padres. }\end{array}$ & & & \\
$\begin{array}{l}\text { - Atendiendo a la profesión del padre. } \\
\text { - Atendiendo a la profesión de la } \\
\text { madre. }\end{array}$ & 187.734 & .430 & $\mathrm{H}^{0}$ \\
$\begin{array}{l}\text { Entre nivel educativo y nivel socio- } \\
\text { económico de los padres. }\end{array}$ & 142.679 & .023 & $\mathrm{H}^{1}$ \\
\hline
\end{tabular}




\section{Conclusiones}

Los resultados obtenidos en este trabajo, nos permite concluir la influencia que variables socio-familiares tienen en el abandono universitario. Son de destacar problemáticas vinculadas a enfermedad en el estudiante o en algún familiar, fallecimiento, tipo de vivienda (vivir alejado de la ciudad, uso del transporte público, residir fuera del hogar familiar) y dificultades económicas (dependencia de beca, incorporación al mercado laboral, estudiantes obligados a simultanear estudios, estudios con trabajo, no actividad de padre).

Una vez identificadas las problemáticas socio-familiares más frecuentes en los estudiantes, otra conclusión importante que nos aporta este trabajo, en concordancia con trabajos de investigación previos, es la relación entre riesgo de abandono y determinadas características de la muestra tales como el sexo, y el nivel socioeconómico de las madres, que está íntimamente relacionado con su nivel educativo.

Un resultado significativo es que es más frecuente el abandono por parte de las mujeres, $72 \%$ de la muestra; presentando a su vez, mayor presencia de problemáticas socio-familiares durante su transcurso universitario. Algunas de las problemáticas que resultan más significativas son "nacimiento de un nuevo miembro de la familia" y "divorcio de los padres" como específicas de su sexo. Entre las problemáticas propias del sexo hombre destacan "simultanear trabajos con estudios".

Estos resultados son concluyentes con los trabajos realizados por Severiens y ten Dam (2012). Estos autores constatan que la presencia de factores socio-familiares influyen de forma diferente entre mujeres y hombres. Las mujeres abandonan por factores personales tales como el nacimiento de los hijos y dificultades familiares; mientras que los hombres desertan a causa de la inserción al mundo laboral.

En relación a la variable nivel socioeconómico observamos diferencias significativas entre riesgo de abandono universitario y profesión actual de las madres. La elevada cifra de madres amas de casa, 33.7\% y en desempleo $10.8 \%$, son características que influyen negativamente en el abandono universitario de los estudiantes. Conforme con Lehmann (2007); Castaño y otros (2008) y García y Adrogué (2015) observamos cómo las familias de estrato medio-bajo tienen mayor riesgo de desertar.

Solo el $26 \%$ de la muestra señala la no presencia de problemáticas socio-familiares. Destacando su desmotivación hacia la carrera (con un 
$11.83 \%$ ) y en menor proporción $(4.73 \%)$ su preferencia por otra institución o titulación.

Así, podemos afirmar que la presencia de problemáticas socio-familiares influye en el abandono del estudiante, pero éstas a su vez vienen acompañadas de otras variables internas al sujeto tales como motivación, engagement, etc. y de la organización concreta a la que se vincula: clima organizativo, cultura docente, etc... Estos resultados son concluyentes con las investigaciones de Cabrera y otros (2006a); Tejedor y GarcíaValcárcel (2007); González y otros (2007); Elías (2008); Bethencourt y otros (2008), en lo que también se ha podido comprobar que los estudiantes con riesgo de abandono, se ven afectados por una multiplicidad de variables (personales, familiares, institucionales y sociales) que son determinantes en su proceso educativo.

Por tanto, podemos concluir que el abandono es el resultado de un conjunto de factores que puestos en interacción desencadenan situaciones de riesgo; alimentando finalmente la decisión de abandonar o continuar los estudios.

En síntesis y para concluir, los resultados obtenidos hacen ver la necesidad de plantear líneas de actuación convergentes que con un carácter prospectivo eviten la deserción tanto a nivel macro como meso estructural. A nivel macroestructural, es el momento de que la universidad, retome su misión como institución socialmente responsable en diferentes ámbitos de su actividad; a nivel meso se hace necesario implantar políticas de permanencia que se acerquen a las problemáticas que viven los estudiantes durante su desarrollo académico, tales como, mejorar los canales de información, ofrecer mejor y más información acerca de los programas que la institución oferta; permitir la movilidad de los estudiantes entre programas educativos; desarrollar programas de asesoramiento sobre estrategias de aprendizaje, etc. En definitiva, realizar un ejercicio de acompañamiento que indague a nivel micro en las dificultades a las que los estudiantes se enfrentan. La potenciación de las funciones orientadoras y tutoriales en distintos momentos de la vida académica de los estudiantes es un área que hay que estimular para generar una universidad inclusiva. 


\section{Referencias bibliográficas}

Aguilera, M.N., y Jiménez, V.E. (2012). Factores de deserción universitaria en el primer curso de las carreras de Trabajo Social y Lengua Inglesa en las Facultades de Humanidades y Ciencias de la Educación y de Lenguas Vivas de la Universidad Evangélica de Paraguay. Revista Internacional de Investigación en Ciencias Sociales, 8 (2), 197-205.

Alonso-Sanz, A., Iglesias Martínez, M.J., y Lozano Cabezas, I. (2015). La conciliación estudiantil-familiar y el proceso de enseñanza-aprendizaje en Educación Superior: un estudio cualitativo. Educatio Siglo XXI, 33 (3), 223-244. doi: http://dx.doi. org/10.6018/j/241001.

Álvarez, P.R., y López, D. (2011). El absentismo en la enseñanza universitaria: un obstáculo para la participación y el trabajo autónomo del alumnado. Bordón. Revista de Pedagogía, 63 (3), 43-56.

Belloc, F., Maruotti, A., y Petrella, L. (2011) How individual characteristics affect university students drop-out: a semiparamentric mixed-effects model for an Italian case study. Journal of Applied Statistics, 38 (10), 2225-2239. doi: 10.1080/02664763.2010.545373.

Bernardo, A., Cerezo, R., Rodríguez-Muñíz, L. J., Núñez, J. C., Tuero, E., y Esteban, M. (2015). Predicción del abandono universitario: variables explicativas y medidas de prevención. Revista Fuentes, 16, 63-84. Recuperado de: https://ojs.publius.us.es/ojs/ index.php/fuentes/article/view/2363.

Bethencourt, J., Cabrera, L., Hernández, J., Álvarez, P., y González, M. (2008). Variables psicológicas y educativas en el abandono universitario. Revista Electrónica de Investigación Psicoeducativa, 6 (3), 603-622.

Bronfenbrenner, U. (1987). La ecología del desarrollo humano: experimentos en entornos naturales y diseñados. Barcelona: Paidós.

Cabrera, L., Bethencourt, J. T., González, M., y Álvarez Pérez, P. (2006a). Un estudio transversal retrospectivo sobre prolongación y abandono de estudios universitarios. RELIEVE, 12 (1), 105-127. Recuperado de: http://www.uv.es/RELIEVE/v12n1/RELIEVEv12n1_1.htm.

Cabrera, L., Bethencourt, J. T, Álvarez, P., y González, M. (2006b). El problema del abandono de los estudios universitarios. RELIEVE, 12 (2), 171-203. Recuperado de: http:// www.uv.es/RELIEVE/v12n2/RELIEVEv12n2_1.htm.

Casquero, T., y Navarro, M.L. (2010). Determinantes del abandono escolar temprano en España: Un análisis por género. Revista de Educación (Número extraordinario), 191-223.

Castaño, E., Gallón, S., Gómez, K., y Vásquez, J. (2008). Análisis de los factores asociados a la deserción estudiantil en la Educación Superior: un estudio de caso. Revista de Educación, 345, 255-280.

Clerici, R., Giraldo, A. y Meggiolario, S. (2015). The determinants of academic outcomes in a competing risks approack: evidence from Italy. Studies in Higher Education, 40 (9), 1535-1549. doi: 10.1080/03075079.2013.878835

Elías, M. (2008). Los abandonos universitarios: retos ante el Espacio Europeo de Educación Superior. Estudios sobre Educación, 15, 101-121.

García, A., y Adrogué, C. (2015). Abandono de los estudios universitarios: dimensión, 
factores asociados y desafíos para la política pública. Revista fuentes, 16, 85-106. doi: http://dx.doi.org/10.12795/revistafuentes.2015.i16.04.

González, M., Álvarez, P., Cabrera, L., y Bethencourt, J. (2007). El abandono de los estudios universitarios: factores determinantes y medidas preventivas. Revista Española de Pedagogía, 236, 71-86.

Gury, N. (2011). Dropping out of higher education in France: a micro-economic approach using survival analysis. Education Economic, 19 (1), 51-64. doi: 10.1080/09645290902796357.

Heublein, U. (2014). Student Drop-out from German Higher Education Institutions. European Journal of Education, 49 (4), 497-513. doi: 10.1111/ejed.12097.

Lassibille, G., y Navarro Gómez, L. (2008). Why do higher education students drop out? Evidence from Spain. Education Economics, 16 (1), 89-105. doi: 10.1080/09645290701523267.

Lehmann, W. (2007). "I just didn't feel like I fi t in": The role of habitus in university dropdrout. Canadian Journal of Higher Education, 37 (2), 89-110.

MECD (2015). Datos básicos del sistema universitario español: curso 2013-2014. Recuperado de http://www.mecd.gob.es/prensa-mecd/dms/mecd/prensa-mecd/ actualidad/2014/02/20140213-datos-univer/datos-cifras-13-14.pdf

Moncada Mora, L. (2014). La integración académica de los estudiantes universitarios como factor determinante del abandono de corto plazo. Un análisis en el sistema de educación superior a distancia del Ecuador. RIED, 17 (2), 173-196.

Naval, C., y Ruiz-Corbella, M. (2012). Aproximación a la responsabilidad social universitaria: la respuesta de la universidad a la sociedad. Bordón, 64 (3), 103-115.

Nora, A. (2001). The depiction of significant others in Tinto's "Rights of Passage": A reconceptualization of the influence of family and community in the persistence process. Journal of College Student Retention, 3 (1), 41-56.

OCDE (2013). Panorama de la educación 2013: Indicadores de la OCDE. Recuperado de http://www.oecd.org/edu/Panorama\%20de\%20la\%20educacion\%202013.pdf.

Patrick, M.E., Shulenberg, J.E., y O'Malley, P.M. (2016). High school substance use as a predictor of collage attendance, completion, and dropout: a national multicohort longitudinal study. Youth \& Society, 48 (3), 425-447. doi: 10.1177/0044118X135089861.

Rojas Betancur, M. (2008). La deserción estudiantil en la Universidad de Ibagué: la perspectiva de los "desertores". Revista Virtual Universidad Católica del Norte, 9, 72-83.

Roso-Bas, F., Pades Jiménez, A., y García-Buades, E. (2016). Emotional variables, dropout and academic perfomance in Spanish nursing students. Nurse Education Today, 37, 53-58. doi: 10.1016/j.nedt.2015.11.021.

Schmitt y Santos (2013). Modelo ecológico del abandono estudiantil en la educación superior: una propuesta metodológica orientada a la construcción de una tesis. En J. Arriaga (coord.) III Conferencia latinoamericana sobre el abandono en la educación superior Ilevada a cabo en Universidad Nacional Autónoma de México (UNAM).

Severiens, S., y Ten Dam, G. (2012). Leaving college: a gender comparison in male and female-dominated programs. Research in Higher Education, 53, (4) 453-457. doi: http://dx.doi.org/10.1007/s11162-011-9237-0. 
Sistema de Garantía de Calidad de los Títulos Oficiales de La Universidad de Sevilla (SGCTUSE). Aprobado en Consejo de Gobierno con fecha 30/09/2008. Versión 4.

Tejedor, F., y García-Valcárcel, A. (2007). Causas del bajo rendimiento del estudiante universitario (en opinión de los profesores y alumnos). Propuestas de mejora en el marco del EEES. Revista de Educación, 342, 443-473.

Vries, W., León, P., Romero, J.F., y Hernández, I. (2011). ¿Desertores o decepcionados? Distintas causas para abandonar los estudios universitarios. Revista de Educación Superior, XL (160), 29-49.

Whannell, R. (2013). Predictors of Attition and Achievement in a Tertiary Bridging Program. Australian Journal of Adult Learning, 53 (2), 280-301.

\section{Notas}

1 El Sistema de Garantía de la Calidad de la Universidad de Sevilla define el indicador abandono inicial como porcentaje de estudiantes de una cohorte de entrada $\mathrm{C}$ que, sin haberse graduado, no se matriculan en los dos cursos siguientes al de su ingreso. Se considera estudiante de nuevo ingreso todos aquellos que han obtenido plaza a través del proceso de pre-inscripción regulado por la comunidad autónoma en cada uno de los cupos (SGCT, V4: p. 36).

2 Este trabajo forma parte del proyecto de investigación europeo titulado "Students guidance at university for inclusión" (Ref: 526600-LLP-12012-IT-Erasmus-Esin) financiado dentro del programa Lifelong learning programme. Call for proposals: EAC/27/11. Erasmus programme. Erasmus multilateral projects. 
